\title{
Finanzas Solidarias para el desarrollo de Mercados Solidarios. La experiencia de las Ferias de la Universidad Nacional de Quilmes
}

(2010-2012)

\author{
Solidarity Finance for the development of Solidarity Markets. The \\ experience of the Fairs of the National University of Quilmes
}

(2010-2012)

ARTÍCULO DE EXTENSIÓN

\author{
Christian Arnaiz \\ Universidad Nacional de Quilmes, Argentina. \\ christianarnaiz@yahoo.com.ar

\section{Santiago Errecalde} \\ Universidad Nacional de Quilmes, Argentina. \\ santiagoerrecalde@gmail.com

\section{Emiliano Recalde \\ Universidad Nacional de Quilmes, Argentina. emilianorecalde@yahoo.com.ar}

\begin{abstract}
Resumen
El trabajo presenta y analiza una experiencia de construcción de mercados y finanzas solidarias desarrollada desde el Proyecto Universitario "Construyendo Redes Emprendedoras en Economía Social" (CREES), del Observatorio del Sur de la Economía Social y Solidaria del Departamento de Economía y Administración de la Universidad Nacional de Quilmes (UNQ), de forma articulada con la Mesa de Promoción de la Economía Social y Solidaria, entre el año 2010 y 2012.

Palabras claves: mercados solidarios; consumo; finanzas solidarias.
\end{abstract}

\begin{abstract}
The paper presents and analyzes the experience of building markets and solidarity finance developed from the University Project Building Entrepreneurial Networks in Social Economy of the Southern Observatory of Social and Solidarity Economy of the Department of Economics and Administration of the National University of Quilmes ( UNQ), in conjunction with the Social and Solidarity Economy Promotion Board, between 2010 and 2012.
\end{abstract}

Key Words: Solidarity markets; consumption; solidarity finance. 


\section{Introducción: Origen de la experiencia}

El presente trabajo busca dar cuenta de la experiencia que llevamos adelante desde el Proyecto Universitario Construyendo Redes Emprendedoras en Economía Social (CREES), asentado en la Universidad Nacional de Quilmes (UNQ). Este, asimismo, se dio en el marco de la Mesa de Promoción de la Economía Social y Solidaria (MePESS) y ha tenido la finalidad de dar respuesta a los problemas de comercialización sobre los productos de la Economía Social y Solidaria (ESS), manifestados tanto por los referentes de las organizaciones sociales -con las que se ha trabajo en el territorio- como por los propios productores asociados a las mismas.

El Proyecto CREES nació en el 2006 como una estrategia de Voluntariado Universitario con la intención de trabajar problemáticas de las organizaciones del territorio que abordaban diferentes formas (espacios de contención, formación, encuentro) y distintas herramientas (microcrédito, emprendimientos) para enfrentar la problemática del trabajo en Quilmes, Varela y Berazategui. Ese mismo año, se ofertó en la UNQ la materia "Teoría y Práctica en ESS" a cargo de Rodolfo Pastore. En la actualidad, CREES ${ }^{1}$ articula en el marco del Observatorio del Sur de la ESS actividades de docencia, extensión, investigación e incubación universitaria para el desarrollo, promoción y fortalecimiento de la socioeconomía.

\footnotetext{
${ }^{1}$ Para más información se recomienda visitar el sitio web: www.observatorioess.org.ar.
} 
Programa de Extensión Universitaria CREES + ICOTEA. 7 proyectos de extensión universitaria
Observatorio del sur de la ESS

(unidad académica)

Programa Universitario de Incubación Social (SEU)
Investigación aplicada al desarrollo de mercados y empresas sociales

Desarrollo de nodos de consumo en territorio

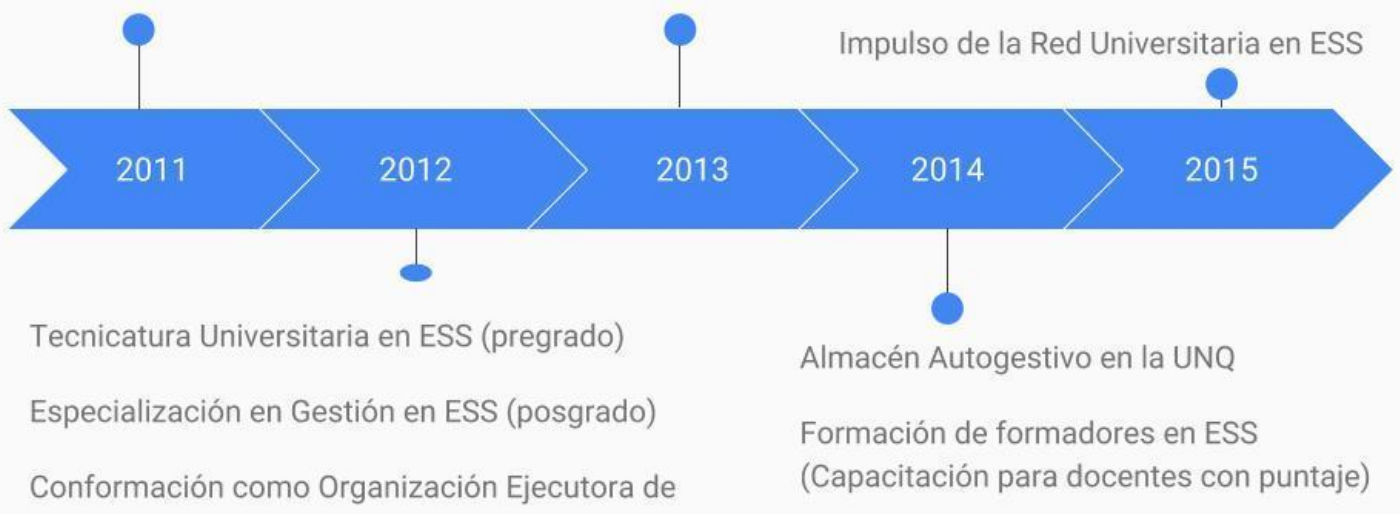

Microcrédito del Ministerio de Desarrollo Social

Gráfico I: Evolución Proyecto CREES serie 2006-2010 (Errecalde, 2017).

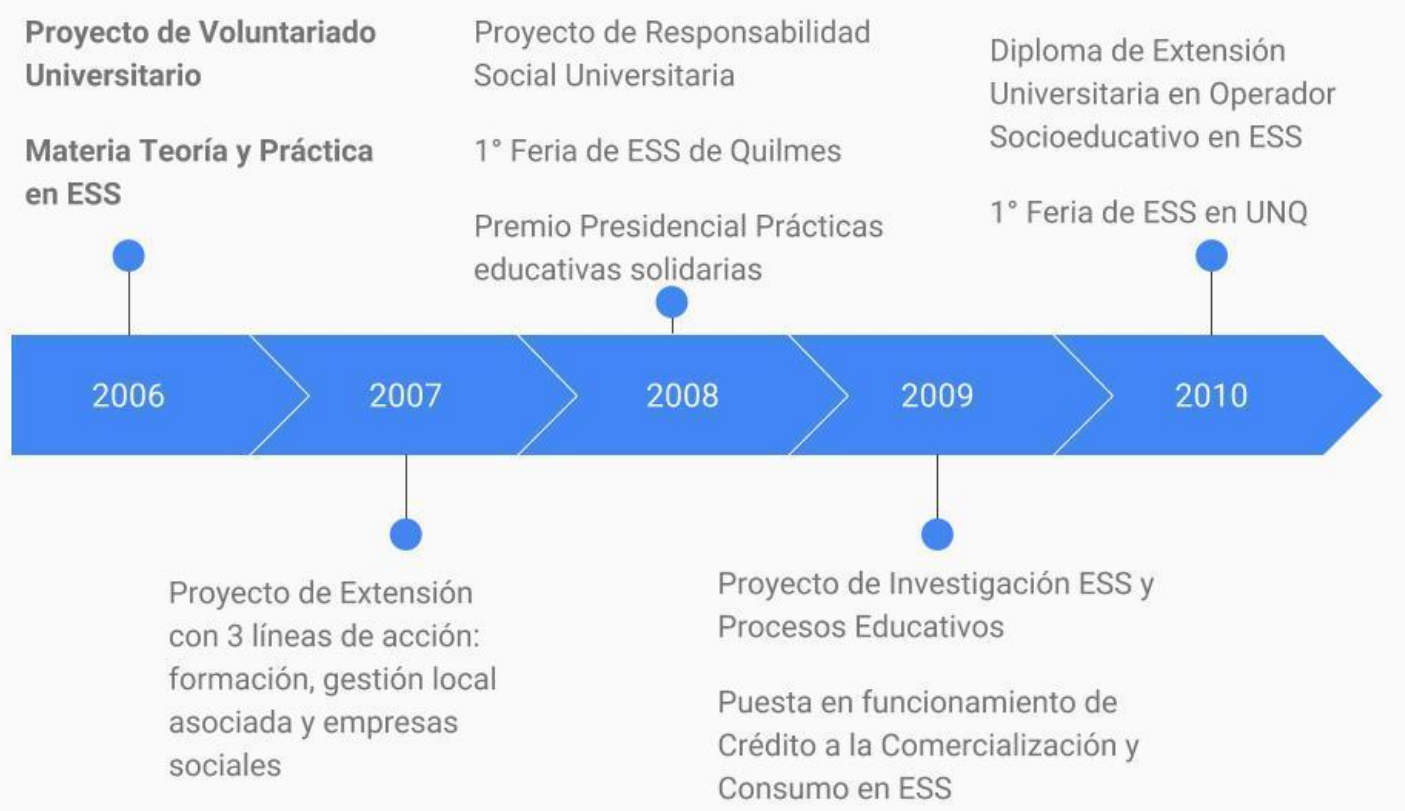

Gráfico II: Evolución Proyecto CREES serie 2011-2015 (Errecalde, 2017) 
La Red Mesa de Promoción de la Economía Social y Solidaria (MePESS) se conformó a partir de un proceso iniciado en el año 2006 por organizaciones sociales, productores, entidades educativas, cientifico-técnicas y estatales. ${ }^{2}$ Tiene entre sus líneas de acción estratégica ${ }^{3}$ la del fortalecimiento económico de los actores que integran el campo. Su finalidad es contrarrestar las tendencias negativas del sistema capitalista hoy hegemónico -en términos de generación de desigualdades, de fomento de "formas de sociabilidad empobrecidas" y de explotación creciente de los recursos naturales (Souza Santos y Rodríguez, 2007, p. 40)- desarrollando estrategias que tiendan al buen-vivir. ${ }^{4}$

Los productores asociados a las organizaciones integrantes de MePESS encuentran diversas dificultades al momento de alcanzar la sostenibilidad socioeconómica de sus emprendimientos productivos. Problemas de acceso a espacios regulares de venta (de costos elevados y con requisitos que no contemplan las características de su producción ni su identidad), la dificultad de ofrecer sus productos en el espacio público (donde la policía puede decomisar su mercancía) y la alta competencia a la que se ven sometidos por su orientación a sectores donde se registra una saturación de la oferta (para los productos que ofrecen, en términos de calidad y precio). A estas dificultades se suma la dispersión de las organizaciones en el territorio que no permite presentarse como un sector de importancia frente a las autoridades del gobierno local ${ }^{5}$ para facilitar los procesos de comercialización (actualmente sigue siendo un desafío aunque con mejores perspectivas).

De este trabajo articulado surgen diferentes preguntas y problemáticas. En este trabajo nos proponemos reflexionar en torno a alguna de ellas: ¿cómo desarrollar herramientas que contribuyan a la sostenibilidad socioeconómica de las organizaciones y

\footnotetext{
2 Organizaciones que se vinculan con productores por espacio territorial a través de microcrédito (MOCAR, EMAC, Colectivo de a pie); instituciones que participan a partir de la oferta de la formación en oficios y para la producción de bienes y servicios o en ESS (CFP 406 de Quilmes, Proyecto CREES - Universidad Nacional de Quilmes); Organizaciones o redes de emprendimientos (Neo-Nos Estamos Organizando; Miel Para Todos; LlajtaMaini) y Políticas Públicas vinculadas a la promoción del autoempleo, trabajo autogestivo (Municipio de Quilmes).

${ }^{3}$ Las siguientes son las líneas de acción estratégicas impulsadas desde un comienzo desde MePESS: 1. Fortalecimiento económico; 2. Representación y confluencia política; 3. Construcción colectiva de conocimientos.

4 Tomamos la idea de buen-vivir porque es la forma tomada por las organizaciones. Reconocemos una multiplicidad de interpretaciones en torno al "Buen-vivir", entre ellas, la del "Plan Nacional Buen-Vivir 2013-2017" de Ecuador (2013, p. 13): "El buen vivir es la forma de vida que permite la felicidad y la permanencia de la diversidad cultural y ambiental en armonía, igualdad, equidad y solidaridad. No es buscar opulencia ni el crecimiento económico infinito". Para ampliar este debate ver las posturas del Sumaq Kawsay y Sumaq Qamaña, ambos planteados desde las cosmovisiones quechua y aymara, y planteadas en las Constituciones de Ecuador y Bolivia, relacionadas a las diferentes formas de entender la economía, sus diversas representaciones y los distintos mercados.

${ }^{5}$ Entre 2012 y 2015 existió en el Municipio de Quilmes un programa de Economía Social y Solidaria que es parte de MePESS y con el que se trabajó de forma participativa en el desarrollo de espacios de comercialización y regulaciones locales. A partir de 2016 con el cambio de signo político del gobierno local, el programa se encuentra en reformulación.
} 
emprendimientos productivos de la ESS? ¿Pueden éstas contribuir a la consolidación de circuitos alternativos de intercambio? ¿Puede de allí pensarse la construcción de mercados solidarios?

En esta dirección, nos proponemos reflexionar sobre las ferias que hemos realizado y las experiencias de intercambio llevadas adelante que han vinculado productores y consumidores mediante la creación y el fortalecimiento de Sistemas de Intercambio Solidarios (SIS) ${ }^{6}$ que benefician tanto a unos -garantizándoles sus medios productivoscomo a los otros -asegurándoles el acceso a los medios de consumo requeridos al buenvivir de las personas (Mance, 2013, p. 1). En particular nos enfocamos en las Ferias realizadas en la UNQ en los años 2010, 2011 y 2012 donde se aplicó un Sistema de Crédito Solidario a partir del uso de una moneda social.

\section{La construcción de un Mercado Solidario (MS) desde una experiencia de Red}

Antes de adentrarnos en la descripción del mencionado dispositivo de Finanzas Solidarias, se considera conveniente mencionar los aspectos que caracterizan al espacio de comercialización en cuestión como un "Mercado Solidario".

En este sentido, la feria como mercado solidario es un espacio donde confluyen el proceso social de producción y el proceso social de circulación. Parte del supuesto de que existen prácticas que ayudan a visualizar el "carácter no monolítico del sistema institucional vigente" (Coraggio, 2011, p. 13). ${ }^{7}$ La conjunción de "Mercado" y "solidario" presenta esa tensión entre el intercambio mercantil monetario y la construcción del reconocimiento recíproco entre personas que garantizan la reproducción de la vida.

Como lo indican Plasencia y Orzi, "el mercado puede ser mirado como un mecanismo de coordinación de múltiples iniciativas autónomamente dirigidas, para expresar necesidades y requerimientos productivos, o para organizar la producción de bienes y servicios útiles para el consumo o la producción, pero no es el único posible" (2007, p. 2). Desde nuestra óptica, el mercado constituye una institución social que da forma a las actividades económicas orientadas al intercambio y es el espacio donde

\footnotetext{
${ }^{6}$ Al respecto se puede citar: "Los Sistemas de Intercambio Solidario son formas organizativas de economía solidaria que integran transacciones monetarias y no-monetarias con la finalidad de asegurar los medios de consumo requeridos al buen-vivir de las personas y medios productivos para la sustentabilidad de emprendimientos solidarios" (Mance, 2013).

7 Para el enfoque sustantivo de la economía, el análisis positivo crítico de la realidad no puede separarse de lo propositivo basado en otro concepto de la racionalidad económica. Esto implica reconocer el carácter no monolítico del sistema institucional vigente, la existencia de proyectos y prácticas contradictorias entre las cuales se perfilan las que llamamos de economía social y solidaria" (Coraggio, 2011).
} 
diversos actores pujan por las formas de organizar la circulación de los satisfactores. Entonces, los intercambios que se realizan en el mercado solidario "con una lógica en la que la búsqueda de ventajas económicas particulares" se expresan "en el marco de consideraciones morales, que limitan el campo de las acciones aceptables de modo que nadie pueda resultar afectado en las condiciones de reproducción de su vida" (pp. 5-6). Por ello, la construcción de un mercado solidario debe contemplar determinadas características y prácticas que puedan atender y potenciar las necesidades de intercambio tanto de productores como de consumidores.

Partimos desde una propuesta de construcción conjunta de la oferta y la demanda. Es decir, buscamos tensionar el supuesto de que la oferta crea su propia demanda para trabajar en los puntos de encuentro y desencuentro de productores y consumidores. Desde nuestra experiencia, resulta tan importante trabajar en la organización de lo que se ofrece como en la organización de lo que se demanda y en las condiciones en que se efectúan los intercambios.

Al respecto, la experiencia de realización de ferias ha buscado promover espacios que propicien tanto el control social de los espacios de comercialización, como un espíritu participativo y democrático en las instancias de decisión que los llevan adelante. En este sentido podemos observar 4 puntos claves:

\section{A. Construcción del marco en que se desarrollan los intercambios}

La propuesta de la Feria supone la construcción de un mercado solidario al mismo tiempo que se desarrollan espacios de encuentro, formación, esparcimiento y construcción de sentidos. Intenta hacer visible las relaciones humanas detrás de los procesos de producción, la diversidad de experiencias y perspectivas que integran la ESS y los valores y principios que enmarcan las acciones impulsadas por las organizaciones asociativas autogestionadas.

\section{B. Complementación de la oferta}

Al realizar la primera feria en la UNQ, se garantizó espacios para la producción local canalizada a través de las organizaciones integrantes de MePESS, pero, a su vez, se buscó complementar la oferta. La complementación de oferta consiste en la invitación a organizaciones y productores de la ESS (por fuera de la red MePESS) a participar en la construcción del mercado solidario con dos finalidades: en primer lugar, aumentar la diversidad de productos y servicios ofrecidos (regionalizando una oferta que se presente como más atractiva para los consumidores); y en segundo lugar, mejorar el intercambio de experiencias y trayectorias entre productores y organizadores.

\section{Construcción y participación democrática del mercado}


Se impulsan estrategias para que todos y todas se involucren en la gestión del MS, en la construcción del objetivo común y en la toma de decisiones, con la premisa de "ir trazando ritmos y ámbitos adecuados de institucionalización de la participación" (Coraggio, 1991, p. 9). En este sentido, se promueven dos espacios de gestión de la feria: las reuniones de la red y las pre-ferias.

Las reuniones de la red son un espacio de participación representativa. Es decir, miembros llevan la voz de las organizaciones al plenario de la red.

Las pre-ferias son encuentros que se desarrollan con anterioridad a la puesta en funcionamiento del MS como un espacio de decisión ampliado y de participación en el que productores, representantes de las organizaciones de MePESS y organizaciones invitadas se encuentran para compartir el recorrido realizado, (re)conocernos como trabajadores/trabajadoras y tratar dos tipos de cuestiones: por un lado, aquellas cuestiones operativas vinculadas a la puesta en funcionamiento de la feria (horarios, estética, gastos, iluminación y otras cuestiones) y; por otro lado, se utiliza el encuentro para trabajar sobre el sentido de la construcción de "Mercados Solidarios" para el desarrollo de la ESS.

\section{Desarrollo de estrategias para involucrar a los consumidores}

En particular, a partir del desarrollo de un sistema de crédito solidario que busca involucrar a consumidores en estrategias organizadas de compra involucrándolos en relaciones comerciales diferentes y ayudando a la visualización de la magnitud de la fuerza del consumo en el circuito económico.

\section{Generando la propia demanda: la necesidad de ganar concurrencia en los espacios de feria y el surgimiento del Crédito a la Comercialización Solidaria (CCS) ${ }^{8}$}

De anteriores procesos de construcción de mercados y ferias hemos sacado algunas conclusiones: a) construir el mercado en lugares de alta circulación de personas y b) propiciar instrumentos que faciliten y traccionen la demanda. A partir del segundo hecho mencionado, fue que decidimos impulsar un dispositivo que permita financiar el consumo en las ferias por medio de la creación de una moneda social que garantice el intercambio y la comercialización. Es así que surge el Crédito a la Comercialización Solidaria (CCS) ${ }^{9}$.

\footnotetext{
${ }^{8}$ Un primer acercamiento y presentación de perspectivas de esta experiencia se puede encontrar en Arnaiz, C., Pastore, R., Recalde, E. y Silva, C (2011) "En el camino de la construcción de las finanzas solidarias: una experiencia de crédito a la comercialización solidaria".

${ }^{9}$ El CCS tiene como fuerte antecedente la experiencia del Banco de Palmas de Brasil y otras experiencias de intercambio con monedas alternativas.
} 
El CCS es una estrategia de Finanzas Solidarias. Como tal, propone discutir el rol de la moneda como consenso social en el camino por democratizar el acceso al dinero y poner las finanzas al servicio de las necesidades de toda la población (Muñoz, 2007, p. 217). De esta manera, se concibe al CCS como parte de los servicios de intermediación que no se enfocan en la reproducción del capital y por ello, se encuentran en la transición hacia otra economía y otro tipo de sociedad.

El sistema de crédito tiene como objetivo la construcción de lazos de cooperación entre la oferta organizada en los mercados solidarios y las organizaciones populares que logran nuclear consumidores, promoviendo y fortaleciendo otras prácticas de producción, distribución y circulación.

El CCS se realiza a través de un Bono a la Comercialización Solidaria (BCS), una moneda social, ${ }^{10}$ que sirve para comprar bienes y servicios en la feria y caduca al finalizar la misma. En tanto moneda, cumple las funciones de instrumento de crédito y de medio de pago. La implementación a través de una moneda social impide el atesoramiento, dinamiza el intercambio y valora elementos que no son estimados en el mercado capitalista: la relación y ponderación de los productores, el fomento de otras formas organizativas y la integración social (Plasencia y Orzi, 2007).

El CCS consiste en una estrategia de financiamiento que busca impulsar el desarrollo y fortalecimiento de la ESS en uno de los principales cuellos de botella del sector, la comercialización y el desarrollo de mercados solidarios. En efecto el CCS es un instrumento de financiamiento e incentivo a la comercialización y consumo de productos de la ESS, articulando organizaciones que por su trabajo territorial se encuentran vinculadas en diversas de redes de la socioeconomía (Arnaiz, et al. 2011).

Para la realización del CCS, se requiere contar con algún tipo de financiamiento que permita funcionar como un fondo rotatorio puesto al servicio del consumo en los mercados solidarios o ferias.

La operatoria del CCS es la siguiente. En los días previos a la realización de la feria en la que se dispuso la utilización del CCS se ofrece crédito a las organizaciones de la red, a las que fueron invitadas a la feria y a los trabajadores de la universidad. Los mismos solicitarán una cantidad de crédito según lo que hayan relevado entre sus miembros y allegados. El día de realización de la feria -al comienzo de la misma- los referentes de cada una de las organizaciones recibirán los créditos solicitados en la forma de BCS, que a su vez reparten entre sus miembros para que compren en la feria.

10 Acerca de la moneda social, Claudia Bisaggio Soares (2009) indica dos significados recurrentes: 1) "medio de intercambio" alternativo o complementario, capaz de generar mejores condiciones de vida a los participantes; y/o 2) práctica de reinvención de la economía, anhelando reconstruirla según pautas responsables y participativas, de forma integrada con las otras esferas de la vida". 
Luego, cada organización recauda lo prestado a sus integrantes y lo devuelve a MePESS en cuatro cuotas mensuales, consecutivas, iguales y sin interés. Los productores organizados, al finalizar la jornada, cambian los bonos por dinero en efectivo y se les retiene el $5 \%$ del total de sus ventas en bonos para cubrir distintos tipos de gastos vinculados tanto a la organización de la feria como a la implementación del CCS.

\begin{tabular}{|c|c|c|c|c|}
\hline Feria & Octubre 2010 & Mayo 2011 & Noviembre 2011 & Octubre 2012 \\
\hline $\begin{array}{l}\text { CCS solicitado en } \\
\text { pesos }\end{array}$ & 55.327 & 122.330 & 30.340 & 55.050 \\
\hline $\begin{array}{l}\text { Ventas totales } \\
\text { registradas en pesos }\end{array}$ & 76.000 & 170.000 & 55.000 & 84.000 \\
\hline $\begin{array}{l}\text { Productores } \\
\text { participantes (oferta) }\end{array}$ & 118 & 120 & 58 & 62 \\
\hline $\begin{array}{l}\text { Organizaciones } \\
\text { tomadoras de crédito }\end{array}$ & 15 & 18 & 14 & 12 \\
\hline Origen de los fondos & $\begin{array}{c}\text { Fuerza Solidaria } \\
\text { Cospe }\end{array}$ & $\begin{array}{c}\text { Fuerza Solidaria } \\
\text { MePESS }\end{array}$ & MePESS & Fuerza Solidaria \\
\hline
\end{tabular}

Tabla 1. Crédito al Consumo Solidario 2010-2012 (elaboración propia).

\section{Consideraciones finales}

Pensar en la sostenibilidad socioeconómica implica dos dimensiones: la sostenibilidad de los emprendimientos y organizaciones asociativas autogestionadas y la de los dispositivos que se desarrollan de forma colectiva como las ferias en tanto mercados solidarios y el sistema de crédito solidario.

El objetivo amplio del Mercado Solidario en tanto estrategia orientada a la reproducción ampliada requiere de mecanismos que dinamicen los intercambios, integren valores y fomente mecanismos de construcción y evaluación democrática.

Como vimos, el CCS se implementa a través de una moneda social que se distribuye, circula y se oxida durante el desarrollo de la feria. La obtención de los fondos, los costos de la operatoria, la organización del mecanismo y el seguimiento de la cartera (préstamos y devoluciones) se asume de forma colectiva por los actores de la red que desarrollan estrategias para la obtención de fondos (presentación de proyectos a entidades de financiamiento, aportes de fondos propios) y para cubrir los costos de la 
operatoria (con horas de trabajo para promover, recuperar y seguir la evolución del crédito; y obteniendo u aportando dinero para el pago de gastos bancarios e intereses).

De la implementación del CCS se desprenden cinco elementos fundamentales: a) fomenta procesos organizativos colectivos al implementarse sólo a través de organizaciones; b) fortalece a las organizaciones y productores de la ESS al incorporar sus productos y servicios en el mercado solidario (ayudando a superar uno de los principales obstáculos para su desarrollo); c) permite a los consumidores obtener bienes y servicios en el mercado solidario pagándolos en cuatro cuotas sin interés e integrándolos en una relación comercial diferente que no es sólo monetaria; d) articula estrategias de financiamiento de forma creativa e innovadora hacia la construcción de finanzas solidarias construyendo vínculos entre las distintas esferas del proceso económico; y e) dinamiza los intercambios aumentando las ventas en la feria.

\section{Referencias Bibliográficas}

Arnaiz, C.; Martínez M.; Silva C.; Errecalde, S.; Recalde, E (2011). La Universidad Nacional de Quilmes y la extensión. Desarrollo de tecnologías sociales para la promoción de Otra Economía: la implementación del Crédito a la Comercialización Solidaria. Recuperado de:

http://www.academia.edu/22039304/La_Universidad_Nacional_de_Quilmes_y_la_extensi \%C3\%B3n._Desarrollo_de_tecnolog\%C3\%ADas_sociales_para_la_promoci\%C3\%B3n_de _Otra_Econom\%C3\%ADa_la_implementaci\%C3\%B3n_del_Cr\%C3\%A9dito_a_la_Comerci alizaci\%C3\%B3n_Solidaria

Bissagio Soares, C. (2009). Moneda Social. En Cattani, Antonio; Coraggio, J. L.; y Laville J.L. (org.). Diccionario de la otra economía. Buenos Aires. Universidad Nacional de General Sarmiento: Altamira y CLACSO.

Coraggio, J. L. (1991). Las dos corrientes de descentralización en América Latina. En Ciudades sin rumbo. Investigación urbana y proyecto popular. Quito: Edición Ciudad-SIAP.

Coraggio, J. L. (2011). Principios, prácticas e instituciones de la economía social y solidaria. En Acosta, A. y Martínez, E. (eds.). El trabajo antes que el capital. Quito: AbyaYala. 
Errecalde, S. (2016). Proyecto Construyendo Redes Emprendedoras en Economía Social y Solidaria. Jornadas de actividades de Consejo de Planeación Regional de la Educación Superior, 24 de abril, Universidad Austral.

Sabaté F.; Muñoz, R. y Ozomek, S. (2004). Introducción. Finanzas y economía social, modalidades en el manejo de recursos solidarios. Buenos Aires: OSDE Altamira.

Mance, E. A. (2013). Sistemas de Intercambio Solidario. En Seminario Internacional Economía Popular y Solidaria "Rol de la Economía Popular y Solidaria y su aporte en el Sistema Económico Social y Solidario- Instituto Nacional de Economía Popular y SolidariaMIES, Ecuador, Quito.

Melo Lisboa, A. (2004). Mercados solidarios. En Cattani, A. D. (comp.). La otra economía. Buenos Aires: Fundación OSDE.

Meyer, N. (2012). Finanzas solidarias y democratización del dinero. En revista del Centro Cultural de la Cooperación, 14/15. Recuperado de: http://www.centrocultural.coop/revista/1415/finanzas-solidarias-y-democratizacion-deldinero

Muñoz, R. (2007). Finanzas para la economía social. Dinero, finanzas y la otra economía. ¿Qué estrategias y alternativas existen? Cartilla $n^{\circ} 1$. Maestría en Economía Social, Instituto del Conurbano, Universidad Nacional de General Sarmiento.

Muñoz, R. (2009). Finanzas Solidarias. En: Cattani, D, Coraggio, J. L, Laville, J. L. Diccionario de la otra economía: lecturas sobre economía social. Buenos Aires: Ed. Altamira.

Pastore, R. (2010). Un panorama del resurgimiento de la economía social y solidaria en Argentina. En Revista de Ciencias Sociales, 2 (18).

Plasencia, A. y Orzi, R. (2007). En torno a los conceptos de mercados solidarios y moneda social. En Moneda Social y Mercados Solidarios. Potencial emancipador y pedagógico de los sistemas monetarios alternativos. Buenos Aires: Ediciones CICCUS.

Singer, P. (2004). La economía solidaria. En Cattani, A. D. (comp.). La otra economía. Buenos Aires: Fundación OSDE. 
Santos, B. de S.; Rodríguez, C. (2012). Para ampliar el canon de la producción. En De las dualidades a las ecologías, Red Boliviana de Mujeres Transformando la Economía (REMTE), Abril, La Paz- Bolivia. 